\title{
Spinal dumbbell-shaped epidural cavernous hemangioma (CM): report of nine surgical cases and literature review
}

\author{
Liang Zhang ${ }^{1 \dagger}$, Zhifeng Zhang ${ }^{1 \dagger}$, Wuyang Yang ${ }^{2}$, Jifeng Shang ${ }^{1}$, Wenqing $\mathrm{Jia}^{1 *}$, Jun Yang ${ }^{1}$ and Yulun Xu
}

\begin{abstract}
Background: Spinal dumbbell-shaped epidural cavernous malformation (CM) is a rare, hypervascular entity frequently misdiagnosed for other lesions, leading to unexpected intraoperative bleeding and suboptimal resection. Our study aims to elucidate the demographics, management strategy, and outcome of this vascular disease.

Methods: Retrospective review of patients seen in Beijing Tiantan Hospital with symptomatic dumbbell-shaped epidural CM from 2008 to 2013. All lesions were pathologically confirmed after resection. The clinical manifestations, radiographic features, and treatment modalities of these cases were analyzed.

Results: We included 9 consecutive patients. Average age was $58 \pm 12$ years (range: 34-79 years), with 66.7\% male. Locations of the CMs were: thoracic $(n=7,77.8 \%)$, cervical $(n=1,11.1 \%)$, and cervicothoracic junction $(n=1,11.1 \%)$. Only one case presented with acute manifestations while others experienced chronic progressive spinal cord symptoms. The initial clinical diagnoses were: schwannoma $(n=6,66.7 \%)$, cavernous hemangioma (CM) $(n=1,11$. $1 \%)$, meningioma $(n=1,11.1 \%)$, and angioma $(n=1,11.1 \%)$. Total resection was achieved in six patients $(66.7 \%)$, and partial resection in the other three patients (33.3\%). Average intraoperative blood loss was $400 \pm 300 \mathrm{ml}$ (range: 100-1000 ml). During an average follow-up of $71 \pm 21$ months (range: 29-94 months), excellent outcome was achieved in seven cases (77.8\%), one partially improved (11.1\%), and one deteriorated (11.1\%). No patients experienced recurrence of symptoms.

Conclusions: Spinal dumbbell-shaped epidural CM is a benign vascular malformation that should be differentiated from other dumbbell-shaped lesions. Accurate preoperative diagnose is challenging as no specific radiographic marker has been established. Total surgical resection should be recommended.
\end{abstract}

Keywords: Cavernous hemangiomas, Spinal cord, Epidural, Dumbbell-shaped, Imaging features, Prognosis

\section{Background}

Spinal hemangiomas, or spinal cavernous malformations $(\mathrm{CM})$, are a rare albeit benign vascular lesion that usually of intra-osseous origin with occasional extension into the epidural space. However, epidural CM without any bony involvement is rarely seen, and a dumbbell-shaped CM may be formed after extra-foraminal extensions under exceedingly rare conditions.

\footnotetext{
* Correspondence: coffeemd@163.com

${ }^{\dagger}$ Equal contributors

${ }^{1}$ Beijing Neurosurgical Institute, Beijing Tiantan Hospital, Capital Medical University, No. 6, Tiantan Xili, Beijing, People's Republic of China

Full list of author information is available at the end of the article
}

Owing to their scarcity, only 11 cases to our knowledge have been reported to date [1-9]. These lesions often morphologically mimic dumbbell-shaped tumors and purported high rate of misdiagnosis before surgical intervention [1]. It is imperative to realize that accurate diagnosis is critical for these types of lesions given that the surgical techniques and outcomes are distinct from other dumbbell-shaped neoplasms, and intraoperative hemorrhage and the clinical outcomes may be formidable considering their hypervascularity. This study is undertaken to analyze the radiological features, surgical management and evaluate the surgical outcomes of nine dumbbell-shaped epidural CM. 


\section{Methods}

A retrospective study was conducted after the consent of our Institutional Review Board (IRB) of Beijing Tiantan Hospital. Patients presenting with symptomatic spinal dumbbell-shaped epidural CM between June 2008 and September 2013 were included. The baseline data of the patients that including age, gender, clinical presentations, spinal radiological features, procedural characteristics, and surgical outcomes was electronically retrieved and reviewed.

When surgical intervention was considered after careful clinical evaluation and spinal cord magnetic resonance imaging (MRI) examination, the procedure was performed through a posterior approach in a lateral position under general anesthesia. The extent of resection was defined as total, subtotal, or partial. Total resection is defined as extirpation of both the intraspinal and extraforaminal portion of the lesion.

All the patients were routinely followed at the outpatient clinics for 3 and 6 months after discharge, and were assessed by telephone or outpatient visit every year subsequently. Follow-up images were obtained in all patients. Neurological outcomes were evaluated using the Modified McCormick Scale (MMS) [10]. A favorable functional outcome was defined as a MMS of 1 or 2 (neurologically intact or mild deficit), and a poor prognosis was defined as a MMS of 3, 4 or 5 (moderate deficit, severe disability or paraplegia).

\section{Results}

\section{Baseline characteristics}

Nine eligible patients were included with a mean age of 58 years (range: $34-79)$, with six (66.7\%) male patients. Clinical course progression before presentation was mostly chronic except for one patient who presented with acute lower limb weakness after two years of chronic course (case two). Two cases were recurrent lesions, one of which underwent surgery four years ago and suffered a recurrent syndrome, and the other patient underwent two procedures at 14 years and 4 years ago, respectively. The preoperative presentations on admission were radiculopathy in 2 patients (22.2\%), and myelopathy in 7 (77.8\%) patients (manifesting as hypoesthesia, lower limb weakness, and urinary or bowel disturbances). The average duration of symptom onset was 11 months, with a range of 1-36 months. Preoperative MMS was as follows: two patients (22.2\%) as MMS I, one (11.1\%) as MMS II, four (44.5\%) as type III, and two (22.2\%) as type IV (Table 1$)$.

\section{MRI characteristics and preoperative diagnosis}

Spinal MRI demonstrated that the lesion location had a thoracic spine predominance $(n=7,77.8 \%)$, followed by cervical $(n=1,11.1 \%)$ and cervicothoracic junction $(n=1$, $11.1 \%)$. The intraspinal part of the lesions were located in the posterior epidural space in four patients $(44.6 \%)$, anterior space in $2(22.2 \%)$ and lateral portion in 3 (33.3\%). All lesions extended through the intervertebral foramen (5 lesions to the left side, 4 lesions to the right side) to present as an intrathoracic mass or cervical mass, which closely mimic the appearance of dumbbell-shaped tumors in coronal and axial view. All cases except for one exhibited isointense signal on T1-weighted images, with the exceptive case demonstrating hypointense signal. All cases had hyper-intensity on T2-weighted MRIs, and all lesions were enhanced after injection of Gadolinium (Fig. 1c). Three patients were revealed with dilated neuroforamen on MRI. On CT scans, all cases featured an isointense signal. Based on these preoperative materials, six lesions were radiologically misinterpreted as schwannomas, one as meningioma, and two as recurrent epidural hemangiomas.

Table 1 Characteristics of Nine Patients with Dumbbell-Shaped Epidural CM

\begin{tabular}{|c|c|c|c|c|c|c|c|c|c|c|c|c|c|c|c|c|}
\hline \multirow{2}{*}{\multicolumn{2}{|c|}{$\begin{array}{l}\text { Age/ } \\
\text { Sex }\end{array}$}} & \multirow[t]{2}{*}{ Symptom } & \multirow[t]{2}{*}{ Duration(months) } & \multirow[t]{2}{*}{ Site } & \multirow{2}{*}{$\begin{array}{l}\text { Initial } \\
\text { Diagnosis }\end{array}$} & \multicolumn{3}{|c|}{ MRI findings } & \multicolumn{3}{|c|}{ Radiologic features } & \multirow{2}{*}{$\begin{array}{l}\text { Intra-op } \\
\text { bleeding } \\
(\mathrm{ml})\end{array}$} & \multirow[t]{2}{*}{ Recurrent } & \multirow{2}{*}{$\begin{array}{l}\text { FU/ } \\
\text { months }\end{array}$} & \multicolumn{2}{|l|}{ MMS } \\
\hline & & & & & & $\mathrm{T} 1 \mathrm{Wl}$ & $\mathrm{T} 2 \mathrm{Wl}$ & $\mathrm{Gd}$ & $\mathrm{CT}$ & $\begin{array}{l}\text { Foramen } \\
\text { Dilation }\end{array}$ & Extension & & & & Pre-op & Last-FU \\
\hline 1 & $55 / \mathrm{M}$ & H/W/U & 1 & T2-4 & Sch & Hypo & Hyper & $\mathrm{HO} / \mathrm{ob}$ & Iso & Yes & $\mathrm{D} / \mathrm{Lt}$ & 200 & No & 94 & III & I \\
\hline 2 & $58 / \mathrm{M}$ & $\mathrm{H} / \mathrm{W} / \mathrm{B}$ & 24 & T3-4 & Sch & Iso & Hyper & $\mathrm{HO} / \mathrm{ob}$ & Iso & No & $\mathrm{D} / \mathrm{Lt}$ & 200 & No & 94 & IV & I \\
\hline 3 & $79 / M$ & $\mathrm{H} / \mathrm{Lp}$ & 4 & T7-8 & CM & Iso & Hyper & HE/ob & Iso & No & $\mathrm{Lt}$ & 200 & Yes & 94 & $\|$ & I \\
\hline 4 & $66 / M$ & H/W/U & 6 & $\mathrm{~T} 2-3$ & Sch & Iso & Hyper & $\mathrm{HO} / \mathrm{ob}$ & Iso & No & $\mathrm{D} / \mathrm{Lt}$ & 1000 & No & 52 & III & I \\
\hline 5 & $63 / M$ & H/W/U & 6 & T3-4 & Sch & Iso & Hyper & $\mathrm{HO} / \mathrm{ob}$ & Iso & No & V/Rt & 100 & No & 90 & III & I \\
\hline 6 & $54 / F$ & $\mathrm{H} / \mathrm{Lp}$ & 36 & C7-T1 & $\mathrm{CM}$ & Iso & Hyper & $\mathrm{HO} / \mathrm{ob}$ & Iso & No & V/Rt & 300 & Yes & 53 & 1 & I \\
\hline 7 & $44 / M$ & $\mathrm{H} / \mathrm{W}$ & 1 & T7-9 & Sch & Iso & Hyper & $\mathrm{HO} / \mathrm{ob}$ & Iso & Yes & Rt & 200 & No & 29 & III & I \\
\hline 8 & $69 / F$ & $\mathrm{H} / \mathrm{W} / \mathrm{Cp}$ & 12 & T5-6 & Men & Iso & Hyper & HE/not ob & Iso & No & $\mathrm{D} / \mathrm{Rt}$ & 800 & No & 66 & IV & III \\
\hline 9 & $34 / F$ & Ap & 12 & C $3-4$ & Sch & Iso & Hyper & HE/ob & Iso & Yes & $L t$ & 600 & No & 67 & I & $\|$ \\
\hline
\end{tabular}



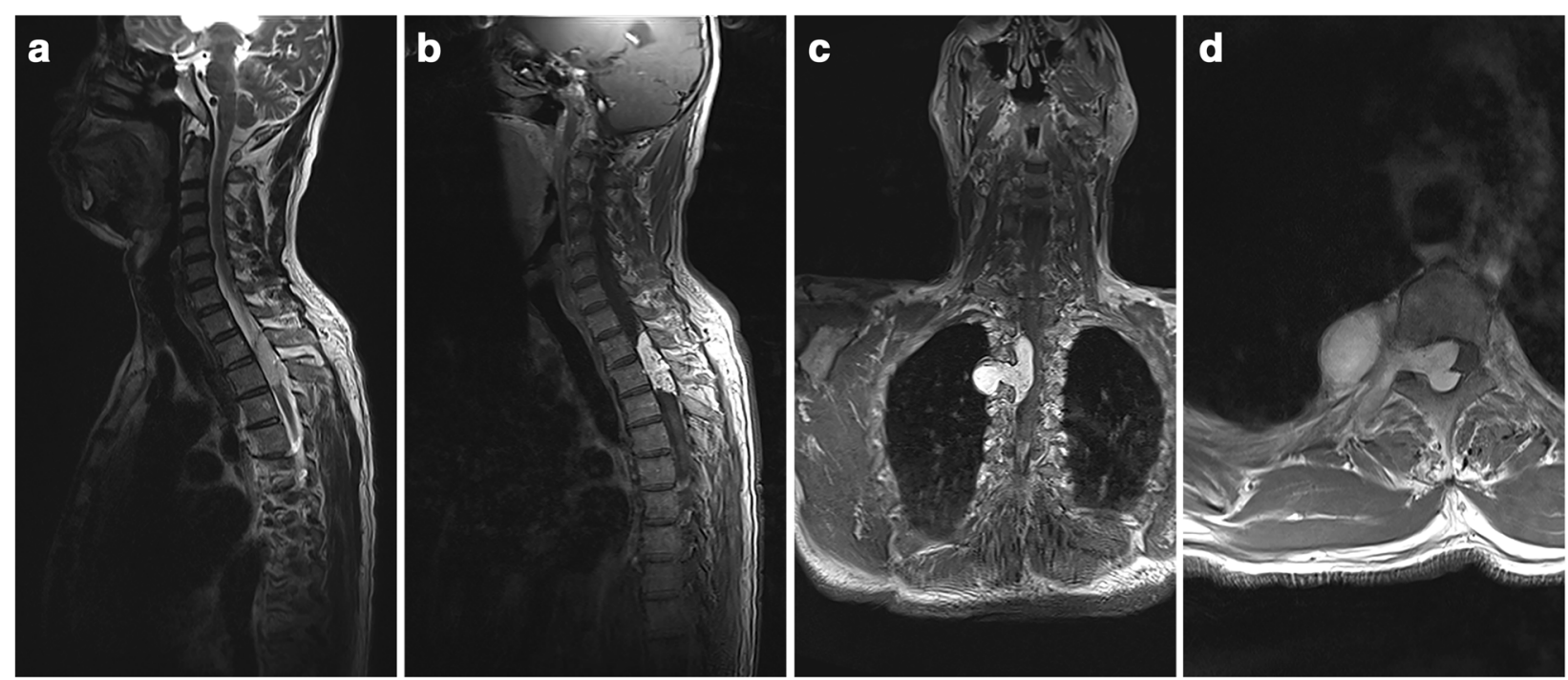

Fig. 1 Preoperative sagittal T2-weighted magnetic resonance images (a) and contrast image (b, c and $\mathbf{d}$ ) shows an epidural lesion from T2 to T3 level, extending through ipsilateral conjunction foramen and with intrathoracic extension (b, $\mathbf{c}$ and $\mathbf{d}$ ). The epidural mass revealed isosignal intensity on T1-weight sequence and hypersignal intensity on T2-weighted sequence, with sagittal, axial and coronal view of homogeneousenhanced after gadolinium injection. The lesions demonstrated isosignal intense in CT scan, without vertebral body erosion and enlargement of the intervertebral foramina

\section{Surgical details intraoperative findings}

All patients were symptomatic before surgery, and a posterior approach in a lateral position under general anesthesia was chosen $[11,12]$. The preoperative decision was planned by conventional posterior approach without thoracoscope or assistance from the cardiothoracic team. Six patients (66.7\%) underwent laminectomy, 1 hemilaminotomy (11.1\%); the two recurrent cases (22.2\%) already underwent previous removal of lamina. A lesion featured dark red or purplish red and well demarcated was revealed in the epidural space after exposure, and was found to be fragile, soft, and bloodrich in the process of dissection. Bipolar was utilized to coagulate the blood vessels and feeding arteries in the surface of the lesions and then shrink the lesions to reduce the hemorrhage volume and facilitate the process of resection. The intraspinal portion was found to be tightly attached to the dura mater; yet the margin of the lesion was easily detected and removed in an en bloc or piecemeal fashion. Notably, significant blood loss maybe encountered intraoperatively due to hypervascular content of the lesion, en bloc method is favored in dealing with intraspinal part for reducing bleeding, and bipolar for coagulation and tight Gelfoam sponge rolls were applied in the process of the resection to terminated bleeding. Two patients experienced extensive bleeding after resection, the bleeding was assumed to originate from the venous plexus around the tumor bed, and tight Gelfoam sponge rolls were used to suppress the cavity with satisfactory control of the bleeding despite rigorous hemostasis. Bone wax may also be used to prevent the hemorrhage when the tumor adhere to the bone or have bone erosion.

Following the total resection of the intraspinal portion, however, all lesions were discovered to have an extraforaminal extension. In five cases, the lesion extended out of the neuroforamen developing into a massive extraspinal portion; of these cases, this portion was subsequently resected through further exposure, and foraminotomy and/or partial medial facetectomy were performed without disrupting the osseous continuity of the interarticularis to avoid spinal stability. What's more, costotransverse was sacrificed in one case to achieve total resection, the extraforaminal portion of the tumor can be detected and removable through the above exposure methods, whereas partial resection was achieved in another case in which the lesion was attached to the vertebral artery, for the remaining two cases only subtotal resection were achieved due to the limitation of the exposure in conventional approach. The tumors usually have a tight adhesion to the nerve root, but can be easily identified after resection of the intraspinal part and further exposure of the neuroforamen, and the nerve root can be easily separated from the tumor without injury. In the 2 recurrent cases, the lesions were found to be adhesive to the surrounding tissues without a clear border. Despite a challenging process, the lesion was completely removed. Average intraoperative blood loss for all patients was $400 \mathrm{ml}$. The pathological findings was showed in Fig. 2. 


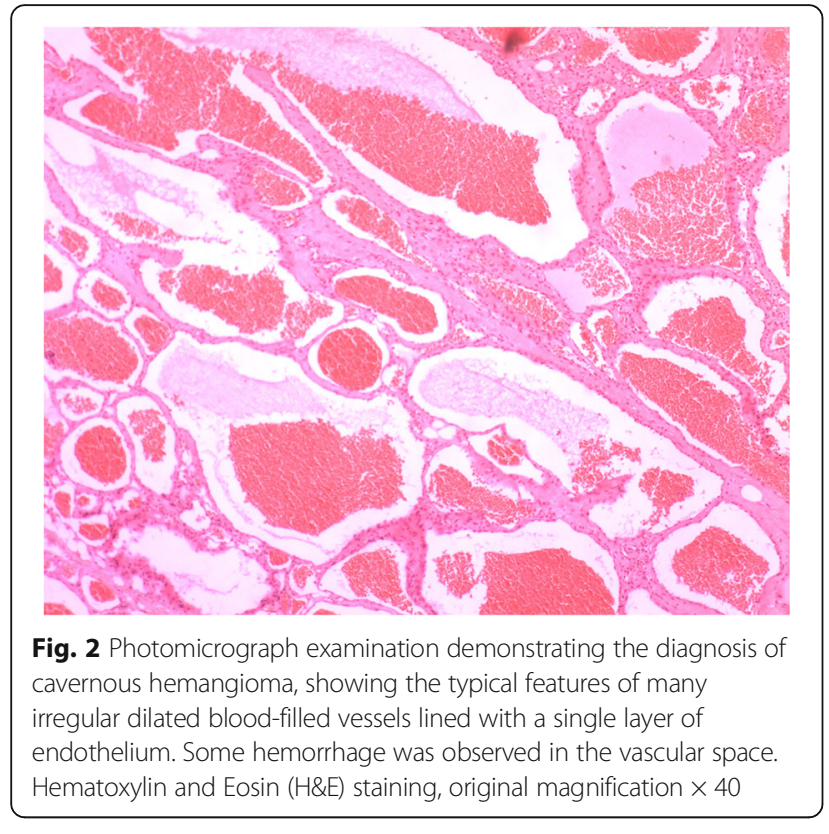

\section{Clinical outcomes and follow-up data}

No procedure-related complication occurred, and all patients had improvement to some degree immediately after operation. Postoperative MRI performed within 7 days and in follow-up period was carefully examined especially for residuals, and no hemorrhage or regrowth of the lesions was found (Fig. 3). Pain has completely resolved in patients presenting with pain, and symptoms such as weakness, sphincter disturbance, and sensory disorders significantly improved during an average follow-up period of 71 months (range: 29-94 months).
However, one case remained functionally unchanged. Majority of these patients experienced no neurological or radiological progression; only one patient with partial removal of the lesion (case 9) experienced neurological progression of muscle atrophy of upper extremity.

\section{Discussion}

Spinal cord CMs are considered to be rare vascular lesions with a congenital nature $[9,13]$. According to classification on the basis of microscopic examination, they are categorized into four types (capillary, cavernous, arteriovenous, or venous), with cavernous type as the major pathological subtype and intramedullary as the most common location in the spinal cord. $[1,12]$ Pure spinal epidural CM accounts for only $4 \%$ of all spinal epidural tumors, and dumbbell-shaped subtype is even more scarce, with only 11 cases been reported in the form of case report [1, 2, 4, 6, 7, 9, 11, 14, 15].

According to published reports as summarized in Table 2, the median age of patients with spinal dumbbell CMs was 50 years, ranging from 23 to 77 years $[1,2]$. Male to female ratio was 7:4 for the 11 reported patients, suggesting a potential predominance of male gender (63.6\%, Table 2). Our cohort consisted of six male $(66.7 \%)$ and three female patients with mean age of 44 years (range 27-56 years). These results are consistent with reported cases in the literature. The most common clinical presentation is myelopathic symptom and nerve root compression, occurring in $72.7 \%$ and $27.3 \%$, respectively (Table 2 ). The duration of symptom onset varied from 1 month to 36 months with a median of 6 months $[2,4,13,14]$. The compression to the spinal

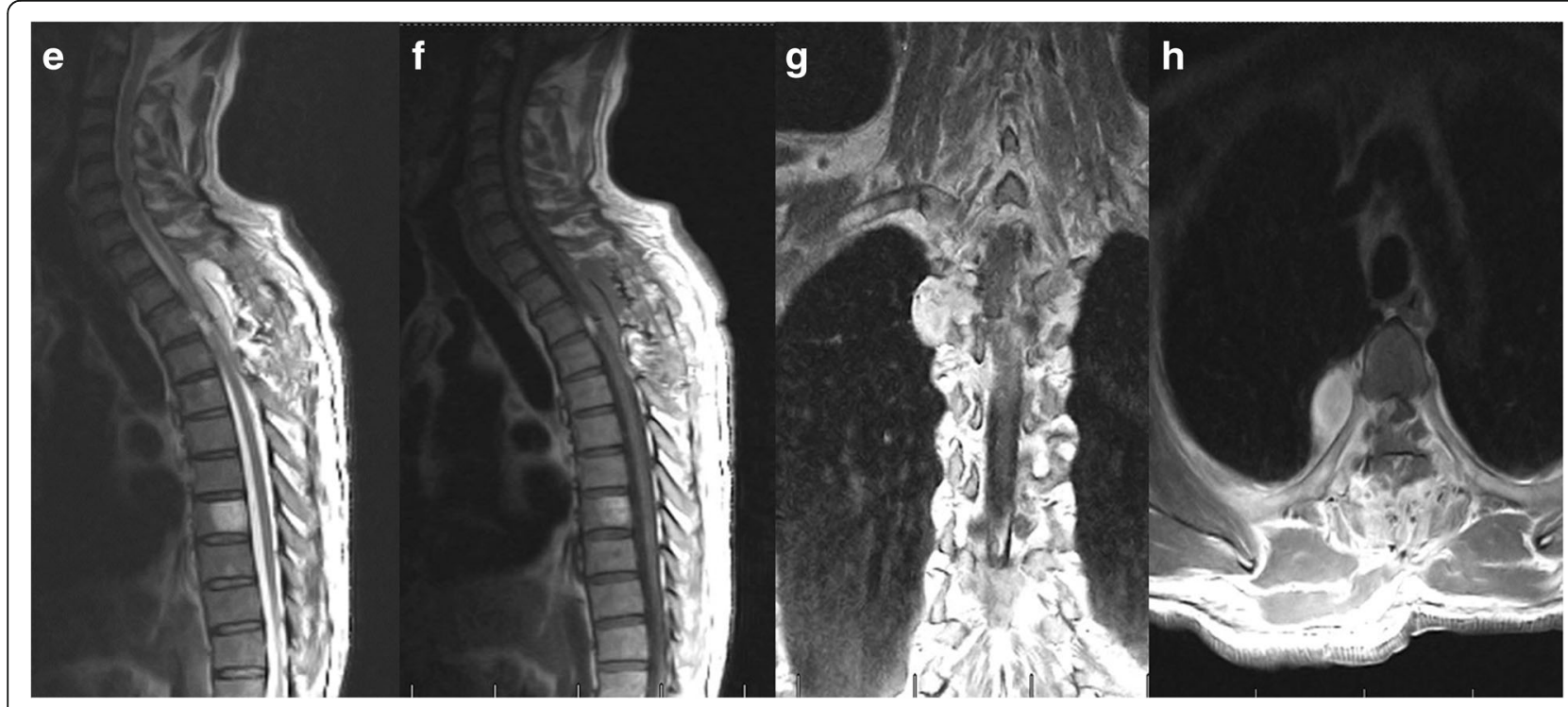

Fig. 3 Postoperative sagittal T2-weighted magnetic resonance images (e) and contrast image (f, $\mathbf{g}$ and $\mathbf{h}$ ) shows partial resection (the intraspinal part was totally removed and the extraforaminal portion was not resected) 


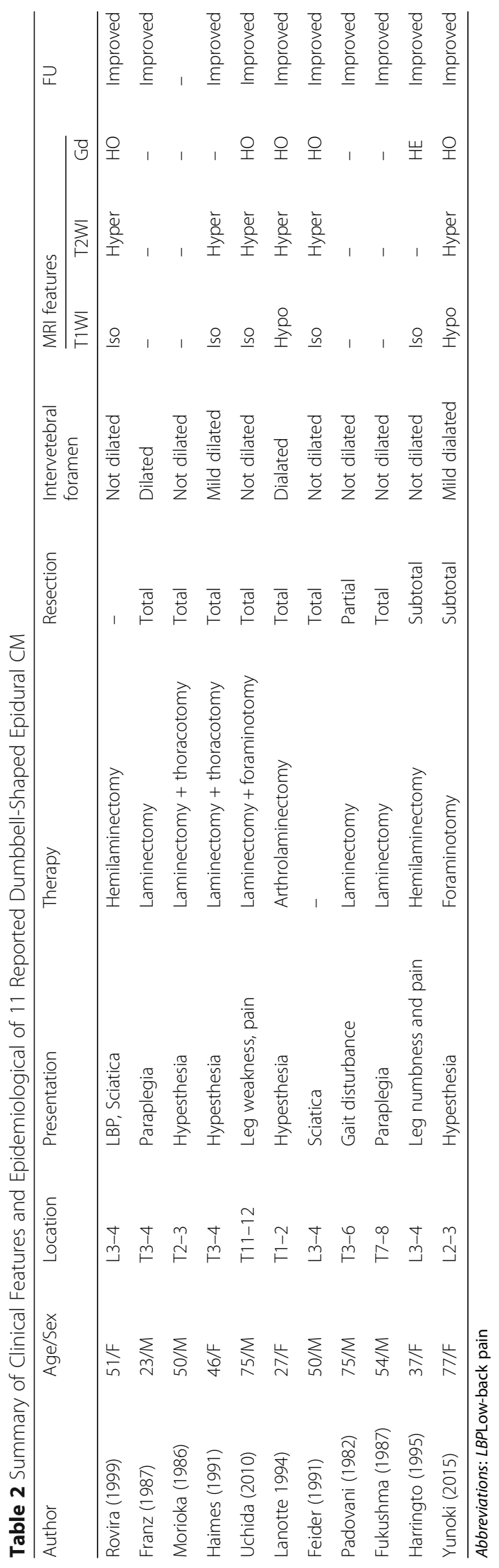


cord can account for myelopathy-related symptoms, whereas nerve root disturbance caused by lesion expansion to the neural foraminal and the mass effect of anterior part of the lesion may account for the symptom of pain $[7,13,16,17]$.

Preoperative MRI is necessary for accurate diagnosis of spinal epidural CM $[1,9,14,18]$. These lesions tend to display iso-signal or low signal on T1WI and high signal on T2WI, with homogeneous enhancement after injection of contrast material, most of which are located in the posterior epidural space and present with myelopathy $[19,20]$. In previous reported cases with specified MRI findings $(n=7)$, six of which showed isosignal on T1WI and high signal on T2WI; additionally, four out of five with contrast material injection demonstrated homogeneous signal enhancement. On rare occasions, epidural CMs may be found to locate in the ventral extradural space and trigger radiculopathy, clinically and radiologically mimicking the presence of disk herniation.[13]On CT scans, these lesions appear as isodensity or slightly hyperdensity signals; however, they are less likely to have a dilated intervertebral foramina, with only $33.3 \%(n=3)$ our series and $27.3 \%(n=3)$ in previous report cases. $[1,2,16]$ Thoracic segment is the predominant location as demonstrated with eight (72.7\%) cases in the thoracic segment in the previous reports and seven $(77.8 \%)$ in our series.

Dumbbell-shaped epidural CMs are almost indistinguishable from that of a neurinoma, neurilemmoma or meningiomas [13, 18, 21, 22]. Morioka et al. proposed to differentiate these tumors and spinal CM by enlargement of intervertebral foramina, suggesting that the latter is rarely likely to cause dilation compared to the former [6]. Conversely, this proposal is contradicted by Lee et al., who found that CM usually leads to a neural foraminal widening [18]. These lesions not only show dilated intervertebral foramina, but also tend to invade the vertebral body, and pedicle of vertebral arch, with heterogeneous enhancement and enhance less than spinal CMs and usually with cystic changes, which is different from spinal epidural CMs. Furthermore, spinal malignant tumors such as lymphoma, metastatic tumors, or sarcoma, which has been reported to constitute $10-40 \%$ of dumbbell-shaped spinal tumors, also tend to lack a dilated intervertebral foramina similar to that of spinal epidural CMs $[13,16,23]$. Therefore, provided with high potentiality of significant intraoperative bleeding, preoperative preparation of worst case-scenario is critical, and the diagnosis of spinal CM is recommended to be prioritized in the differential diagnosis of dumbbell-shaped lesions even when no dilation of intervertebral foramina is found. In our series, excluding the two recurrent cases, six cases were preoperatively diagnosed as schwannomas, with another case diagnosed as meningioma. This result and previous report suggests that the most common preoperative misinterpretation of dumbbell spinal epidural CM was schwannoma.

Complete resection is currently the best option for management of epidural dumbbell-shaped CMs in cases with high risk of rebleeding and progressive neurologic deterioration with acceptable outcome.[12, 14] However, for incompletely resected lesions, even no rebleeding and regrowth occurred in our series, the potential of event occurrence still remains, and radiation therapy has been recommended as an alternative. Sohn et al. observed no growth of lesion at 3-years follow-up after stereotactic radiosurgery for a residual lesion [24]. However, the effect of adjuvant radiosurgery remains to be proven since its long-term benefit is unclear $[1,24]$.

\section{Limitations of study}

This study is a retrospective study and potential biases exist. Given the limited sample size, the conclusion inferred from the results of this single series might be biased and susceptible to random observation. However, this is still the largest series on spinal dumbbell-shaped epidural CM reported to-date.

\section{Conclusions}

Spinal dumbbell-shaped epidural cavernous CM is a rare vascular entity with potential extensive intraoperative bleeding. It is therefore recommended that this pathology should be considered in the differential diagnoses of other paravertebral dumbbell-shaped lesions. According to our experience and reported cases surgical resection may achieve satisfactory outcomes with rigorous and meticulous drive of hemostasis. Although extraforaminal portion is commonly inaccessible and maybe left with residual lesions with conventional laminectomy, no recurrent bleeding or growth of the remaining lesion was observed in the long-term follow-up.

\begin{abstract}
Abbreviations
CM: cavernous malformation; IRB: Institutional Review Board; MMS: Modified McCormick Scale; MRI: magnetic resonance imaging;
\end{abstract}

\section{Acknowledgements}

We thank all the patients who trusted us with their care and all of the physicians who helped in this study.

\section{Funding}

The study was supported by Beijing Municipal Health System High-level Health Technical Personnel Training Program (Grant 2015-3-043) and Beijing Municipal Administration of Hospitals Incubating Program (Grant PX2017005).

\section{Availability of data and materials}

All data generated or analyzed during this study are included in this published article [and its supplementary information files].

\section{Authors' contributions}

LZ, ZZ, JS, and WJ collected and analyzed data regarding the clinical data and neuroimaging. WJ, JY, and YX performed the operation. LZ, and WY 
were major contributor in writing and revising the manuscript. All authors read and approved the final manuscript.

\section{Ethics approval and consent to participate}

All procedures performed in studies involving human participants have received Beijing Tiantan Hospital ethics approval.

\section{Consent for publication}

The study has received all related patients' written informed consent.

\section{Competing interests}

The authors report no conflict of interest.

\section{Author details}

${ }^{1}$ Beijing Neurosurgical Institute, Beijing Tiantan Hospital, Capital Medical University, No. 6, Tiantan Xili, Beijing, People's Republic of China.

2Department of Neurosurgery, Johns Hopkins University School of Medicine, Baltimore, MD, USA.

Received: 8 August 2017 Accepted: 10 December 2017

Published online: 15 January 2018

\section{References}

1. Yunoki M, Suzuki K, Uneda A, Yoshino KA. Case of dumbbell-shaped epidural cavernous angioma in the lumbar spine. Surg Neurol Int. 2015; 6(Suppl 10):S309-12

2. Uchida K, Yayama T, Nakajima H, et al. Microsurgical resection of cavernous haemangioma around the thoracic neuroforamen: a case report. J Orthop Surg (Hong Kong). Dec 2010;18(3):370-3.

3. Talacchi A, Spinnato S, Alessandrini F, luzzolino P, Bricolo A. Radiologic and surgical aspects of pure spinal epidural cavernous angiomas. Report on 5 cases and review of the literature. Surg Neurol. Aug 1999;52(2):198-203.

4. Rovira A, Rovira A, Capellades J, Zauner M, Bella R, Rovira M. Lumba extradural hemangiomas: report of three cases. AJNR Am J Neuroradiol. Jan 1999;20(1):27-31.

5. Padovani R, Tognetti F, Proietti D, Pozzati E, Servadei F. Extrathecal cavernous hemangioma. Surg Neurol. Dec 1982;18(6):463-5.

6. Morioka T, Nakagaki H, Matsushima T, Hasuo K. Dumbbell-shaped spinal epidural cavernous angioma. Surg Neurol. Feb 1986;25(2):142-4.

7. Lanotte M, Massaro F, Faccani G, Forni M, Valentini MC. Dumbbell-shaped spinal epidural cavernous angioma. Case report. Ital J Neurol Sci. Nov 1994; 15(8):429-32.

8. Franz K, Lesoin F, Leys D, Krivosic I, Jomin M. Spinal epidural dumbbellshaped cavernous angioma. Rev Neurol. 1987;143(4):298-300.

9. Feider HK, Yuille DL. An epidural cavernous hemangioma of the spine. AJNR. Am J Neuroradiol. Mar-Apr 1991;12(2):243-4.

10. McCormick PC, Torres R, Post KD, Stein BM. Intramedullary ependymoma of the spinal cord. J Neurosurg. Apr 1990;72(4):523-32.

11. Li TY, YL X, Yang J, Wang J, Wang GH. Primary spinal epidural cavernous hemangioma: clinical features and surgical outcome in 14 cases. J Neurosurg Spine. Jan 2015;22(1):39-46.

12. Zhang L, Yang W, Jia W, et al. Comparison of outcome between surgica and conservative Management of Symptomatic Spinal Cord Cavernous Malformations. Neurosurgery. Apr 2016;78(4):552-61.

13. Shukla D, Rao VS, Rajesh A, Uppin MS, Purohit AK. Lumbar extradural dumbbell cavernous hemangioma: a rare lesion. J Neurosci Rural Pract. Apr 2013:4(2):207-9.

14. Haimes $A B$, Krol G. Dumbbell-shaped spinal cavernous hemangioma: a case report. AJNR. Am J Neuroradiol. Sep-Oct 1991:12(5):1021-2.

15. Fukushima M, Nabeshima Y, Shimazaki K, Hirohata K. Dumbbell-shaped spinal extradural hemangioma. Arch Orthop Trauma Surg. 1987;106(6): 394-6.

16. Isoda H, Takahashi M, Mochizuki T, et al. MRI of dumbbell-shaped spinal tumors. J Comput Assist Tomogr. Jul-Aug 1996;20(4):573-82.

17. Saringer W, Nobauer I, Haberler C, Ungersbock K. Extraforaminal, thoracic, epidural cavernous haemangioma: case report with analysis of magnetic resonance imaging characteristics and review of the literature. Acta Neurochir. Dec 2001;143(12):1293-7.

18. Lee JW, Cho EY, Hong SH, et al. Spinal epidural hemangiomas: various types of MR imaging features with histopathologic correlation. AJNR. Am J Neuroradiol. Aug 2007;28(7):1242-8.
19. Khalatbari MR, Abbassioun K, Amirjmshidi A. Solitary spinal epidural cavernous angioma: report of nine surgically treated cases and review of the literature. Eur Spine J. Mar 2013;22(3):542-7.

20. Gencpinar P, Acikbas SC, Nur BG, et al. Epidural capillary hemangioma: a review of the literature. Clin Neurol Neurosurg. Nov 2014;126:99-102.

21. Jeong WJ, Choi I, Seong HY, Roh SW. Thoracic extradural cavernous hemangioma mimicking a dumbbell-shaped tumor. J Korean Neurosurg Soc. Jul 2015;58(1):72-5.

22. Aoyagi N, Kojima K, Kasai H. Review of spinal epidural cavernous hemangioma. Neurol Med Chir. Oct 2003;43(10):471-5. discussion 476

23. Nakano S, Yoneyama T, Sugimoto T, Wakisaka S. Paramedian transmuscular access to C-3 dumbbell-type neurofibroma without paravertebral muscle dissection from the spinous process or facetectomy. Technical note. J Neurosurg. Jul 2003;99(1 Suppl):121-4.

24. Sohn MJ, Lee DJ, Jeon SR, et al. Spinal radiosurgical treatment for thoracic epidural cavernous hemangioma presenting as radiculomyelopathy: technical case report. Neurosurgery. 2009;64(6):E1202-3.

\section{Submit your next manuscript to BioMed Central and we will help you at every step:}

- We accept pre-submission inquiries

- Our selector tool helps you to find the most relevant journal

- We provide round the clock customer support

- Convenient online submission

- Thorough peer review

- Inclusion in PubMed and all major indexing services

- Maximum visibility for your research

Submit your manuscript at www.biomedcentral.com/submit 